\title{
BIO.I4 - Construction, by rational design, and initial characterization of affinity mutants of Rituximab fragment antibody
}

Larissa Queiroz Pontes ${ }^{1 \star}$; Marcus Rafael Lobo Bezerra'; Marcela Helena Gambim Fonseca²; Marcos Roberto Lourenzoni²; Gilvan Pessoa Furtado ${ }^{2}$.

1UFCe - Universidade Federal do Ceará;

2Fiocruz Ceará.

Introduction: Antibodies are glycoproteins, which consist of two types of domains light and heavy chains, each of which is composed of variable and constant domains. Rituximab is a monoclonal antibody used in the treatment of lymphomas and its mechanism of action is based on the interaction of CD20 membrane protein, expressed on B lymphocytes, guiding them to depletion. Single-chain fragments variable $(\mathrm{scFv})$ are composed by the variable domains of antibody heavy and light chains, linked by a flexible polypeptide, making them easier to manipulate on protein engineering techniques, such as rational design, where amino acids are chosen by in silico structural and energetic analysis and changed by site-directed mutagenesis in order to set a better or new biological function.

Objective: Perform site-directed mutagenesis, based on structural and energetic features, on the variable region of Rituximab, in order to increase its affinity to the CD20 epitope.

Methodology: Rituximab scFv mutants were obtained in vitro by site-directed mutagenesis and their sequences confirmed. The bacterial expression of wide-type (wt) scFv and its mutants was standardized to get soluble proteins. The strategy used accomplishes bacterial strain SHUFFLE and pETSUMO vector on overnight expression at $19^{\circ} \mathrm{C}$. These fragments were confirmed by western blot (WB) nitrocellulose membrane, using $5 \%$ defatted milk on overnight blockage $\left(4^{\circ} \mathrm{C}\right), 1 \mu \mathrm{g}$ of each protein and 1:5000 ratio to protein L-HRP. CD on "far-UV" was accessed to obtain the structural secondary profile of scFv's (wt and mutants) using $250 \mu \mathrm{g} / \mathrm{mL}$ of proteins in phosphate buffer $\mathrm{pH}$ 7.0. A peptide-based ELISA was achieved sensitizing the plate with $1 \mu \mathrm{g} /$ well CD20-epitope peptide overnight at $4^{\circ} \mathrm{C}$, using $150 \mu \mathrm{g} / \mathrm{mL}$ of proteins and 1:1500 ratio of protein L-HRP at 1 hour each at room temperature.

Results: Five Rituximab's scFv variants were constructed and expressed at soluble grade. WB membrane revealed the presence of scFv fragments. CD analysis showed similar profiles among the mutant and wt fragment, characterized by $\beta$-sheet secondary structure. Analysis of ELISA confirmed the capacity of binding to immobilized biotinylated peptide of the CD20 epitope from all the fragments compared (wt and mutants scFv).

Conclusion: The results showed an efficient method of bacterial expression of soluble antibody fragments, which were confirmed by WB. The secondary structure was confirmed by CD and besides the mutations; they remained capable of binding to the epitope, based on ELISA assay. This work avenues quantification affinity based on microscale thermophoresis of antibody fragments, followed by construction of the $\mathrm{scFv}-\mathrm{Fc}$ format, in order to study effector functions of those fragments.

Keywords: Antibody engineering; Site-directed mutagenesis; Rituximab 